\title{
Utility of preoperative 3 Tesla pelvic phased-array multiparametric magnetic resonance imaging in prediction of extracapsular extension and seminal vesicle invasion of prostate cancer and its impact on surgical margin status: Experience at a Canadian academic tertiary care centre
}

\author{
Taehyoung Lee, MD; Jen Hoogenes, PhD; Ian Wright, MD; Edward D. Matsumoto, MD; Bobby Shayegan, MD
}

Department of Surgery, Division of Urology, McMaster University, Hamilton, ON, Canada

Cite as: Can Urol Assoc J 2017;11 (5):E174-8. http://dx.doi.org/10.5489/cuaj.4211

Published online May 9, 2017

\section{Abstract}

Introduction: To evaluate the utility of 3 Tesla (3T) pelvic phasedarray (PPA) multiparametric magnetic resonance imaging (mpMRI) to predict extracapsular extension (ECE) and seminal vesicle invasion (SVI) and its subsequent effect on radical prostatectomy (RP) surgical margin status.

Methods: A retrospective evaluation was conducted of RP patients who underwent preoperative 3T PPA mpMRI (without endorectal coil) based on clinical probability of adverse pathological features. Frequencies, specificity, sensitivity, positive predictive value (PPV), and negative predictive value (NPV) of mpMRI in predicting the status of ECE and SVI were calculated.

Results: Forty-eight consecutive patients were included. Sensitivity, specificity, PPV, and NPV for 3T PPA mpMRI using $T_{2}$-weighted sequences with diffusion-weighted imaging (DWI) and dynamic contrast enhanced (DCE) imaging to predict ECE was 39\%, 56\%, $45 \%$, and $50 \%$, respectively, while SVI prediction was 33\%, 95\%, $50 \%$, and $91 \%$, respectively. Twelve of the 28 cases predicted as being negative for ECE had positive margins, while two of the 20 cases predicted to be positive for ECE had positive margins. Imaging predicted four cases would have SVI, yet two had positive margins, while of the 44 cases predicted as being negative for SVI, four had positive margins.

Conclusions: These findings at our centre suggest that the use of 3T PPA mpMRI using $\mathrm{T}_{2}$-weighted sequences with DWI and DCE in predicting pathological ECE and SVI is of questionable benefit. These mpMRI reports may result in closer dissection of neurovascular bundles and subsequent positive surgical margins. Caution should be exercised when basing intraoperative decisions on mpMRI findings.

\section{Introduction}

Multiple tools for diagnosing and staging prostate cancer (PCa) have been developed and used routinely for patienttailored treatment and management. Imaging modalities, such as transrectal ultrasound (TRUS)-guided biopsy and computed tomography $(\mathrm{CT})$ have assisted in determining the appropriate treatment for patients presenting with PCa. ${ }^{1-3}$ Despite the utility of these tools, they are often limited in their ability to predict extracapsular extension (ECE) of PCa. ${ }^{3}$ Data have shown that $27 \%$ of patients clinically diagnosed with ECE actually have locally confined disease on pathology, while $25-30 \%$ of patients diagnosed with organ-confined disease actually have ECE at final pathology., ${ }^{4,5}$ Magnetic resonance imaging (MRI) has emerged as a promising method to increase the precision of preoperative staging and to predict ECE and seminal vesicle invasion (SVI). Multiple MRI modalities are available, and the literature reports wide ranges of sensitivity and specificity of its ability to predict ECE and SVI despite modality, calling the validity and utility of MRI for preoperative workup into question. 3,6,7 Heidenreich et al stated that multiparametric MRI (mpMRI) with $\mathrm{T}_{2}$-weighted dynamic contrast-enhanced (DCE) with diffusion-weighted imaging (DWI) has excellent sensitivity for detecting Gleason $\geq 7 \mathrm{PCa}$; yet these promising results need further confirmation. The cost-effectiveness of mpMRI and interrater reliability among radiologists is a current concern. ${ }^{8}$ Thus, the ability of mpMRI to detect ECE and SVI has not yet been fully established.

Radical prostatectomy (RP) has emerged as a standard treatment for localized PCa, providing an approximate 10-year progression-free survival rate of approximately $90 \%$ of patients. ${ }^{9}$ The presence of ECE and SVI carries a substantial risk of positive surgical margin rate, compromising postoperative oncological outcomes. It has been reported that 
10-year progression-free probability decreases to $71.4 \%$ for patients with ECE and $37.4 \%$ for patients with SVI. ${ }^{9}$ Accurate staging, precise preoperative selection of PCa cases, and planning of surgical approach are crucial in achieving ideal oncological and functional outcomes. Preoperative imaging that provides adjunctive staging information may assist planning, thus potentially improving postoperative outcomes. ${ }^{10}$

We evaluated the ability of 3Tesla (3T) pelvic-phased array (PPA) mpMRI without endorectal coil to predict ECE and SVI prior to RP. We further assessed the consequent effect that MRI findings had on surgical margin status in a cohort of patients treated by a single, experienced urological oncologist.

\section{Methods}

\section{Study design and participants}

Consecutive patients who underwent MRI prior to RP (robotassisted, laparoscopic, or open approach) by a single, experienced urological oncologist at our academic tertiary care centre from 2009-2013 were eligible for inclusion in this retrospective review. Preoperative 3T PPA mpMRIs were chosen at the discretion of the surgeon based on high clinical probability of adverse pathological features. Indicators of possible ECE and/or SVI included high prostate-specific antigen PSA (PSA $>10 \mathrm{ng} / \mathrm{ml}$ ), low free-to-total ratio $(\mathrm{F} / \mathrm{T}$ ratio $<0.10)$, high PSA density (PSAD >0.15), cT3 (a and/or b) disease, high-grade disease (presence of Gleason 4 or 5 via TRUS biopsy), and high-volume disease (multiple cores with $>50 \%$ involvement). Any combination of the aforementioned indicators, along with comprehensive history and physical examination, prompted MRI investigation in patients with suspected advanced PCa. MRIs were conducted at least six weeks post-biopsy to avoid risk of hemorrhage artifact on $\mathrm{T}_{2}$-weighted images. Given lack of clear guidelines for MRI use in management of $\mathrm{PCa}$, the decision to use preoperative MRI was a clinical one, rather than one based on prescribed parameters.

\section{Test methods}

The 3T PPA mpMRI (Phillips Achieva 3.0T, Amsterdam, Netherlands) with $\mathrm{T}_{2}$-weighted DWI and DCE with spectroscopy for axial, sagittal, and coronal views of the prostate was used, employing a standardized protocol for each patient. All images were read by a genitourinary radiologist, who is the sole expert (>10 years of experience) in reporting on mpMRI of the prostate at our high-volume tertiary care academic centre. He was blinded to each patient's history, including biopsy characteristics. Prostate Imaging Reporting and Data System (PI-RADS) version one was used until the validated version two was released. The RP operative approach, including resection plane and nerve-sparing, was modified by the surgeon based on MRI evidence of ECE and/or SVI presence.

\section{Data analysis}

Tumour stage based on preoperative MRI characteristics was compared to final pathological stage. Specificity, sensitivity, positive predictive value (PPV), and negative predictive value (NPV) of 3T PPA mpMRI in predicting ECE and SVI, as well as the prevalence of ECE and SVI and likelihood ratios were calculated in IBM SPSS v.22 (Armonk, NY, U.S.). Positive surgical margin rates were calculated in all patients as a proxy to estimate surgical and oncological outcomes. The effect of radiological ECE findings on surgical margin status post-RP was examined by comparing positive surgical margin rates between patients with positive or negative preoperative ECE findings.

\section{Results}

Forty-eight patients comprised the study sample (Table 1 ). The mean age was $60.9( \pm 8.3)$ and the mean preoperative PSA score was $9.7( \pm 2.1)$. The majority of patients $(71 \%)$ were classified as intermediate-risk on the D'Amico risk group stratification criteria $^{11}$ (intermediate: stage $\mathrm{T} 2 \mathrm{~b}$ or Gleason score of 7 or PSA level of $>10$ and $\leq 20 \mathrm{ng} / \mathrm{mL}$ ) and most patients had Gleason 7 disease, both at biopsy (71\%) and on surgical pathology (88\%). The majority of patients underwent a minimally invasive RP $(96 \%)$, while $4 \%$ had the open approach.

Table 1. Patient and disease characteristics and subsequent method of surgical intervention

\begin{tabular}{lcc}
\hline Number of participants & & Patients \\
Mean age & & 48 \\
Mean preoperative PSA score & & $60.9 \pm 8.3$ \\
& 6 & $9.7 \pm 2.1$ \\
Gleason score at biopsy & 7 & $12(25 \%)$ \\
& 8 & $34(71 \%)$ \\
& 9 & $1(2 \%)$ \\
Gleason score at RP & 6 & $1(2 \%)$ \\
& 7 & $3(6 \%)$ \\
D'Amico risk stratification & 8 & $42(88 \%)$ \\
& Intermediate & $2(4 \%)$ \\
Surgical method & High & $7(2 \%)$ \\
& Laparoscopic & $32(14 \%)$ \\
\hline PSA: prostate-specific antigen; RP: radical prostatectomy. & $14(29 \%)$ \\
\hline
\end{tabular}


Lee et al.

\section{Predictability of ECE and resultant surgical margin rates}

The mpMRI reports predicted $20(42 \%)$ patients to be positive for ECE, while final pathology revealed that only nine of these patients were positive for ECE. Of the $28(58 \%)$ patients who were predicted to not have ECE based on mpMRI, 14 had positive ECE on pathology. Preoperative 3T PPA mpMRI achieved a sensitivity of $39 \%$ and a specificity of $56 \%$ in predicting ECE on surgical pathology, with a PPV and a NPV of $45 \%$ and $50 \%$, respectively. Of the 20 patients with positive ECE reported on mpMRI, two (10\%) had positive surgical margins. Furthermore, out of the 28 patients predicted to be negative for ECE, 12 (42.9\%) had positive surgical margins on pathology. The prevalence of ECE in the patient sample was $47.9 \%$, with a negative likelihood ratio of 1.09 and a positive likelihood ratio of 0.89 (Fig. 1). Subgroup analysis of patients with Gleason score 7-9 revealed similar findings, with sensitivity of $39.1 \%$ and specificity of $54.5 \%$ for predicting ECE. Further subgroup analysis of patients with low-volume disease (1-2 positive biopsy cores) and high-volume disease $(>2$ positive biopsy cores) revealed sensitivities of $0 \%$ and $47.4 \%$ and specificities of $33.3 \%$ and $68.4 \%$, respectively.

\section{Predictability of seminal vesicle invasion}

The mpMRI reports predicted four (8\%) patients to be positive for SVI. Final pathology showed that two of these patients were positive for SVI. Of the 44 (92\%) patients who were predicted not to have SVI, four had positive SVI on pathology. Preoperative 3T PPA mpMRI achieved a sensitivity of 33\% and a specificity of $95 \%$ in predicting SVI on surgical pathology. The PPV and NPV were $50 \%$ and $91 \%$, respectively.

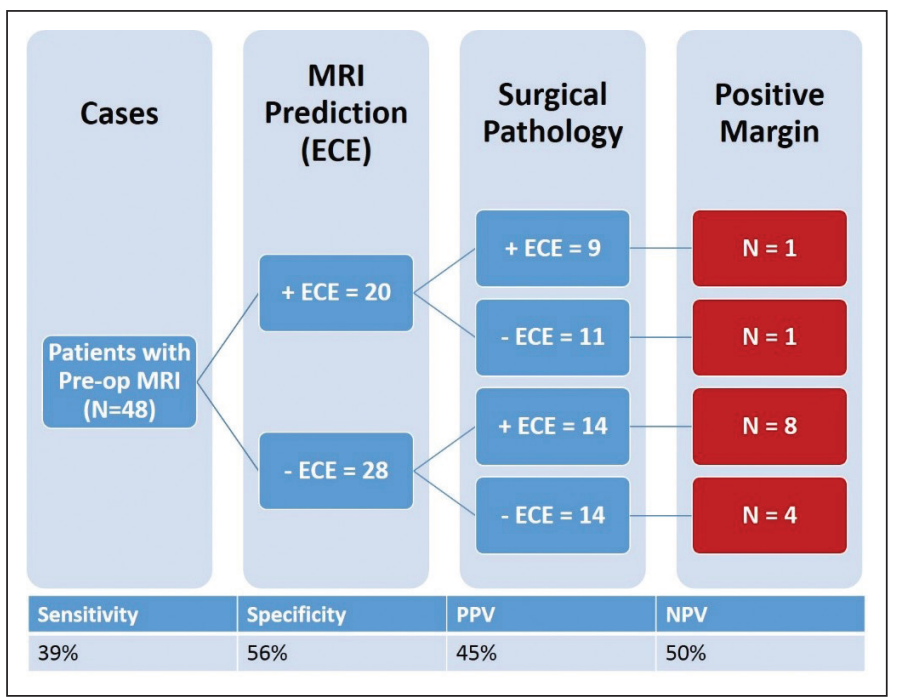

Fig. I. Summary of radiological and pathological extracapsular extension (ECE) and resultant positive margin status in patients who underwent preoperative 3 Tesla pelvic phased-array multiparametric magnetic resonance imaging (MRI). PPV: positive predictive value; NPV: negative predictive value.
Finally, the prevalence of SVI in the sample was $12.5 \%$, while the negative likelihood ratio was 0.70 and the positive likelihood ratio was 7.0 (Fig. 2). Subgroup analysis of patients with Gleason score 7-9 revealed similar findings with sensitivity of $33.3 \%$ and specificity of $94.9 \%$ for predicting SVI. Further subgroup analysis of patients with low-volume disease (1-2 positive biopsy cores) and high-volume disease ( $>2$ positive biopsy cores) revealed sensitivities of $0 \%$ and $50.0 \%$ and specificities of $100 \%$ and $94.1 \%$, respectively.

\section{Discussion}

Our study of 48 patients with moderate- (71\%) to highrisk (14.5\%) PCa demonstrated that the results of a 3T PPA mpMRI should be interpreted with caution during RP planning. The sensitivity and specificity of MRI in predicting ECE were $39 \%$ and $56 \%$, respectively, suggesting that the use of MRI in our sample was not much different than a coin toss. These findings contrasted studies that have reported sensitivity and specificity ranges of $75-78 \%$ and $92-96 \% .^{12-14}$ Evaluation of SVI in our sample showed that although the sensitivity was low at $33 \%$, the specificity was high at $95 \%$, with a PPV of $50 \%$ and a high NPV of $91 \%$. This also contrasts with studies that have shown much higher sensitivity and PPV percentages. ${ }^{6,15}$

The literature suggests that MRI has utility in the workup of $\mathrm{PCa} .{ }^{16-19} \mathrm{Kim}$ et al analyzed 32 preoperative patients using a surface coil and a combined DWI and DCE mpMRI technique at $1.5 \mathrm{~T}$ in predicting the stage, using $\mathrm{RP}$ results as the reference standard. In detecting ECE, the combination of these tests displayed $82.4 \%$ sensitivity, $87.2 \%$ specificity, $70 \% \mathrm{PPV}$, and $93.2 \% \mathrm{NPV}^{20}{ }^{20}$ In 158 patients with clinical

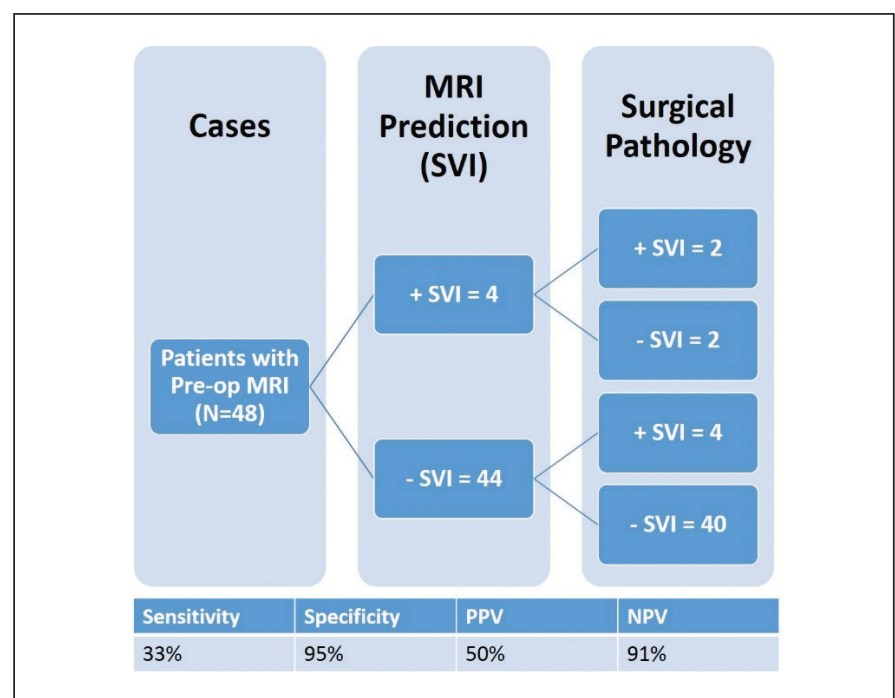

Fig. 2. Summary of radiological and pathological seminal veside invasion (SVI) status in patients who underwent preoperative 3 Tesla pelvic phased-array multiparametric magnetic resonance imaging (MRI). PPV: positive predictive value; NPV: negative predictive value. 
stage T1c disease, Zhang et al analyzed the role of preoperative combined endorectal coil spectroscopic imaging (MRSI) at $1.5 \mathrm{~T}$ to predict the pathologic stage of PCa. The overall accuracy was $80 \%$, while staging accuracy was higher for the smallest tumour volumes $\left(91 \%\right.$ for tumours $<0.5 \mathrm{~cm}^{3}$ vs. $75 \%$ for tumours $>2.0 \mathrm{~cm}^{3}$ ). In the detection of ECE, MRSI had an area under the curve of $0.74 . .^{21}$ In a population of 27 patients considered for RP, Augustin et al compared the accuracy of 3T MRI with the Partin tables predicting pathological stage. In detecting ECE, accuracy was $85.2 \%$, sensitivity was $66.7 \%$, and specificity was $100 \%$. The Spearman's for correlation with ECE was higher for MRI findings (0.780) than for the Partin tables (0.363). They concluded that 3T MRI was significantly more accurate than the Partin tables in predicting the final pathological stage. ${ }^{22}$

Studies using an endorectal coil tend to report better outcomes than those using a surface magnet. The European Association (EAU) guidelines on PCa (2014) state that the use of an endorectal coil can improve staging accuracy at 1.5T, and results obtained at $3 \mathrm{~T}$ are superior to $1.5 \mathrm{~T} \mathrm{f}^{8,23}$ There is a debate as to whether the use of endorectal coils is necessary. Lee and colleagues stated that they are expensive and cause discomfort and potential proctitis and diverticulitis. ${ }^{24}$ Their study showed no difference in MRI staging accuracy between those with the endorectal coil vs. a surface coil. ${ }^{24}$ Further, in a retrospective analysis of 32 patients with moderate- to highrisk $\mathrm{PCa}$, Kim et al reported that surface coil at 1.5T yielded sensitivity and specificity of $83.3 \%$ and $92.3 \%$, respectively, in predicting SVI. ${ }^{20}$ Uncertainty remains regarding the most useful coil and modality of MRI in staging PCa.

The relative ineffectiveness of 3T PPA mpMRI at our centre may attest to the fact that many barriers exist in routinely performing preoperative MRI in Canada due to the government-funded nature of the healthcare system. This contrasts with multitiered payment systems in other countries, where MRI has become part of the standard workup for newly diagnosed PCa patients. ${ }^{25}$ However, Xylinas et al stated that the routine use of MRI for preoperative evaluation of $\mathrm{PCa}$ is controversial, as its high cost might burden the healthcare system, ${ }^{26}$ and D'Amico et al asserted that although MRI findings can add a significant predictive value, it does not justify its routine use. ${ }^{11}$

In our study, a larger proportion of patients for whom MRI predicted absence of ECE experienced subsequent positive surgical margins (42.9\%) compared to those for whom MRI predicted presence of ECE (10\%). The highest number of positive surgical margins was observed in the group with negative radiological ECE results who turned out to have ECE on pathology ( $67 \%$ had positive surgical margins). It is possible that negative radiological ECE findings may have resulted in dissection closer to the prostatic tumour. Due to the small sample size, the generalizability of the ability of $\mathrm{mpMRI}$ to aid in minimizing positive surgical margin status is in question. This study provides insight into the use of mpMRI to assist in surgical planning for RP.

\section{Conclusion}

At our Canadian healthcare centre, the use of 3T PPA mpMRI without endorectal coil for the prediction of ECE and SVI for preoperative planning yielded less than satisfactory results with patients who had high-volume PCa. Our data suggest that caution should be exercised when implementing preoperative MRI to determine ECE and SVI to modify and/or prepare for subsequent operative approach. A large multicentre study of mpMRI will better define the efficacy, utility, and feasibility of the test and will help define the role of MRI in preoperative assessment of PCa.

Competing interests: The authors report no competing personal or financial interests.

This paper has been peer-reviewed.

\section{References}

1. Faria EF, Chapin BF, Muller RL, et al. Radical prostatectomy for locally advanced prostate cancer: Current status. Urology 2015;86:10-15. https://doi.org/10.1016/j.urology.2015.03.012

2. Lowrance WT, Scardino PT. Predictive models for newly diagnosed prostate cancer patients. Rev Urol 2009;11:117-26.

3. Cerantola Y, Valerio M, Kawkabani Marchini A, et al. Can 3T multiparametric magnetic resonance imaging accurately detect prostate cancer extracapsular extension? Can Urol Assoc J 2013;7:E699-703. https://doi.org/10.5489/cuaj.245

4. Han M, Partin AW, Piantadosi S, et al. Era-specific biochemical recurrence-free survival following radical prostatectomy for clinically localized prostate cancer. J Urol 2001;166:416-9. htrps://doi.org/10.1016/ S0022-5347(05)65955-1

5. Cooperberg MR, Lubeck DP, Mehta SS, et al. Time trends in clinical risk stratification for prostate cancer: Implications for outcomes. J Urol 2003;170:S21-7. https://doi.org/10.1097/01. ju.0000095025.03331.c6

6. Somford DM, Hamoen EH, Futterer JJ, et al. The predictive value of endorectal 3 Tesla multiparametric magnetic resonance imaging for extraprostatic extension in patients with low-, intermediate- and high-risk prostate cancer. J Urol 2013;190:1728-34. https://doi.org/10.1016/i.juro.2013.05.021

7. Braitbord IS, Lavery HJ, Nabizada-Pace F, et al. Endorectal magnetic resonance imaging has limited clinical ability to preoperatively predict pT3 prostate cancer. BJU Int 2011;107:1419-24. https://doi.org/10.1111/i.1464-410X.2010.09599.x

8. Heidenreich A, Bastian PJ, Bellmunt J, et al. EAU guidelines on prostate cancer. Part 1: Screening, diagnosis, and local treatment with curative intent-update 2013. Eur Urol 2014;65:124-37. https://doi.org/10.1016/i.eururo.2013.09.046

9. Hull GW, Rabbani F, Abbas F, et al. Cancer control with radical prostatectomy alone in 1000 consecutive patients. J Urol 2002;167:528-34. https://doi.org/10.1016/S0022-5347(01)69079-7

10. Li B, Du Y, Yang H, et al. Magnetic resonance imaging for prostate cancer clinical application. Chin J Cancer Res 2013;25:240-9.

11. D'Amico AV, Desjardin A, Chen MH, et al. Analyzing outcome-based staging for clinically localized adenocarcinoma of the prostate. Cancer 1998;83:2172-80. https://doi.org/10.1002/(SICI) 10970142(19981115)83:10<2172::AID-CNCR16>3.0.C0;2-K

12. Griebling TL, Ozkutlu D, See WA, et al. Prognostic implications of extracapsular extension of lymph node metastases in prostate cancer. Mod Pathol 1997;10:804-9

13. Porcaro $A B$, Borsato $A$, Romano $M$, et al. Accuracy of preoperative endorectal coil magnetic resonance imaging in detecting clinical understaging of localized prostate cancer. World J Urol 2013;31:1245-51. https://doi.org/10.1007/s00345-012-0900-7 
Lee et al.

14. Bloch BN, Genega EM, Costa DN, et al. Prediction of prostate cancer extracapsular extension with high spatial resolution dynamic contrast-enhanced 3T MRI. Eur Radiol 2012;22:2201-10. https://doi.org/10.1007/s00330-012-2475-5

15. Ghafoori $M$, Alavi $M$, Shakiba $M$, et al. The value of prostate MRI with endorectal coil in detecting seminal vesicle involvement in patients with prostate cancer. Iran J Radiol 2015;12:145-56.

16. Bloch BN, Furman-Haran E, Helbich TH, et al. Prostate cancer: Accurate determination of extracapsular extension with high-spatial-resolution dynamic contrast-enhanced and T2-weighted MR imaging — initial results. Radiology 2007;245:176-85. https://doi.org/10.1148/radiol.2451061502

17. Futterer JJ, Heiimink SW, Scheenen TW, et al. Prostate cancer localization with dynamic contrastenhanced MR imaging and proton MR spectroscopic imaging. Radiology 2006;241:449-58. https://doi.org/10.1148/radiol.2412051866

18. Kurhanewicz J, Vigneron D, Carroll P, et al. Multiparametric magnetic resonance imaging in prostate cancer: Present and future. Curr Opin Urol 2008;18:71-7. https://doi.org/10.1097/M0U.0b013e3282f19d01

19. Mazaheri Y, Shukla-Dave A, Hricak H, et al. Prostate cancer: identification with combined diffusion-weighted MR imaging and 3D 1H MR spectroscopic imaging —correlation with pathological findings. Radiology 2008;246:480-8. https://doi.org/10.1148/radiol.2462070368

20. Kim B, Breau RH, Papadatos D, et al. Diagnostic accuracy of surface coil magnetic resonance imaging at 1.5T for local staging of elevated risk prostate cancer. Can Urol Assoc J 2010;4:257-62. https://doi.org/10.5489/cuaj.09103
21. Zhang J, Hricak H, Shukla-Dave A, et al. Clinical stage Tlc prostate cancer: Evaluation with endorectal MR imaging and MR spectroscopic imaging. Radiology 2009;253:425-34. https://doi.org/10.1148/ radiol.2532081390

22. Augustin $H$, Fritz GA, Ehammer $T$, et al. Accuracy of 3Tesla magnetic resonance imaging for the staging of prostate cancer in comparison to the Partin tables. Acta Radiol 2009;50:562-9. https://doi.org/10.1080/02841850902889846

23. Scialpi M, Piscioli I, D'Andrea A. Underestimated role of MRI in EAU guidelines on prostate cancer. Magn Reson Imaging 2014;32:402-3. https://doi.org/10.1016/i.mri.2014.01.002

24. Lee SH, Park KK, Choi KH, et al. Is endorectal coil necessary for the staging of clinically localized prostate cancer? Comparison of non-endorectal vs. endorectal MR imaging. World J Urol 2010;28:667-72. https://doi.org/10.1007/s00345-010-0579-6

25. Klotz KH. MRI and prostate cancer: The next frontier. Can Urol Assoc J 2010;4:227-8. https://doi.org/10.5489/cuai.895

26. Xylinas $E$, Yates DR, Renard-Penna $R$, et al. Role of pelvic phased array magnetic resonance imaging in staging of prostate cancer specifically in patients diagnosed with clinically locally advanced tumours by digital rectal examination. World J Urol 2013;31:881-6. https://doi.org/10.1007/s00345-011-0811-z

Correspondence: Dr. Bobby Shayegan, St. Joseph's Healthcare Hamilton Institute of Urology, Hamilton, 0N, Canada; shayeb@mcmaster.ca 